\title{
SHORT REPORT \\ Season of birth and risk of rotavirus diarrhoea in children aged $<5$ years
}

\author{
C. J. ATCHISON ${ }^{1 *}$, C. C. TAM ${ }^{1}$ AND B. A. LOPMAN ${ }^{1,2}$ \\ ${ }^{1}$ Infectious Diseases Epidemiology Unit, Department of Epidemiology and Population Health, London School \\ of Hygiene and Tropical Medicine, London, UK \\ ${ }^{2}$ Gastrointestinal, Emerging and Zoonotic Infections Department, Centre for Infections, Health Protection \\ Agency, London, UK
}

(Accepted 10 December 2008; first published online 15 January 2009)

\section{SUMMARY}

This study investigates whether a child's risk of rotavirus diarrhoea is associated with season of birth in England and Wales, countries where rotavirus infections are highly seasonal. Poisson regression models were fitted to weekly counts of laboratory-confirmed rotavirus infections from children aged $<5$ years born between 1998 and 2007. In the first year of life, the risk of a laboratory-confirmed rotavirus infection was significantly higher for children born in summer

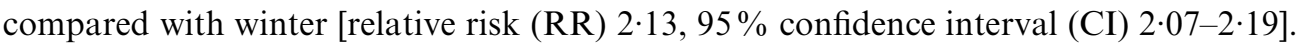
In the second to fifth years of life, the pattern reversed (second year of life: RR $0 \cdot 73,95 \% \mathrm{CI}$ $0 \cdot 71-0 \cdot 75)$. The cumulative risk up to age 5 years remained significantly higher for children born in summer compared with winter due to the increased risk for summer births during their first year of life. Maternal immunity and age-specific levels of exposure to rotavirus could explain our findings.

Key words: Diarrhoea, gastroenteritis, rotavirus, seasonality.

Over 13000 laboratory-confirmed cases of rotavirus diarrhoea are reported in England and Wales each year. The estimated community rate of rotavirus disease is $7 \cdot 1 / 1000$ person-years [1]; in cases of gastroenteritis, rotavirus infection is identified most commonly in children aged $<5$ years [2]. The risk factors associated with rotavirus disease in children in England, as identified by a case-control study, include living in rented council housing, accommodation with $<5$ rooms, contact with another person with gastroenteritis; and in infants, not being exclusively breastfed [3].

\footnotetext{
* Author for correspondence: Dr C. J. Atchison, Infectious Diseases Epidemiology Unit, Department of Epidemiology and Population Health, London School of Hygiene and Tropical Medicine, London WC1E 7HT, UK.

(Email: Christina.Atchison@1shtm.ac.uk)
}

In Europe rotavirus disease exhibits a distinct seasonal pattern, the causes of which are not well understood [4]. A winter/early spring peak in reported cases is typical, but the exact timing of the peak varies between countries. In England and Wales it occurs in late February/early March [5]. Protective immunity to rotavirus is relatively complex, with various factors contributing to immunity: maternal antibodies confer some protection for newborns, neonatal infection is believed to offer partial protection against disease, and previous infections progressively reduce a child's risk of subsequent rotavirus infection and disease [6]. The incidence of clinical disease peaks between ages 6 and 24 months [7].

Given these immunological and seasonal characteristics, we hypothesized that a child's risk of rotavirus diarrhoea depends partly on the child's season 
Table 1. Gender, age and season of birth distribution of reports of laboratory-confirmed rotavirus infections in children aged $0-4$ years in England and Wales (1998-2007)

\begin{tabular}{|c|c|c|c|c|}
\hline & $\begin{array}{l}\text { Reported } \\
\text { cases, } \\
\text { no. }(\%)\end{array}$ & $\begin{array}{l}\text { Rate } \\
\text { (per } 1000 \\
\text { person-years) }\end{array}$ & $\begin{array}{l}\text { Unadjusted } \\
\text { RR }(95 \% \text { CI })\end{array}$ & $P$ value \\
\hline \multicolumn{5}{|l|}{ Gender } \\
\hline Male & $52902(55 \cdot 2)$ & $5 \cdot 9$ & 1 & \\
\hline Female & $42935(44 \cdot 8)$ & $4 \cdot 5$ & $0.77(0.76-0.78)$ & $<0.001$ \\
\hline \multicolumn{5}{|l|}{ Age (yr) } \\
\hline 0 & $45870(47 \cdot 9)$ & $9 \cdot 2$ & 1 & \\
\hline 1 & $35217(36 \cdot 7)$ & $8 \cdot 2$ & $0.88(0.87-0.90)$ & $<0.001$ \\
\hline 2 & $10181(10 \cdot 6)$ & $2 \cdot 8$ & $0 \cdot 30(0 \cdot 29-0 \cdot 31)$ & $<0.001$ \\
\hline 3 & $3347(3 \cdot 5)$ & $1 \cdot 1$ & $0 \cdot 12(0 \cdot 11-0 \cdot 12)$ & $<0.001$ \\
\hline 4 & $1222(1 \cdot 3)$ & $0 \cdot 5$ & $0 \cdot 05(0 \cdot 05-0 \cdot 06)$ & $<0.001$ \\
\hline \multicolumn{5}{|c|}{ Season of birth } \\
\hline Winter & $21435(22 \cdot 4)$ & $4 \cdot 8$ & 1 & \\
\hline Spring & $25081(26 \cdot 2)$ & $5 \cdot 4$ & $1 \cdot 13(1 \cdot 11-1 \cdot 15)$ & $<0 \cdot 001$ \\
\hline Summer & $26240(27 \cdot 4)$ & $5 \cdot 5$ & $1 \cdot 15(1 \cdot 13-1 \cdot 17)$ & $<0.001$ \\
\hline Autumn & $23081(24 \cdot 1)$ & $5 \cdot 0$ & $1.03(1 \cdot 01-1 \cdot 05)$ & $0 \cdot 001$ \\
\hline
\end{tabular}

RR, Relative risk; CI, confidence interval.

of birth. We investigated whether the season of birth is related to the risk of rotavirus diarrhoea in England and Wales.

The Health Protection Agency (HPA) collects reports of laboratory-confirmed rotavirus infections from laboratories across England and Wales. These laboratories receive stool specimens for testing from clinicians seeing patients in hospital or primary healthcare facilities in both urban and rural settings. Most laboratories offer rotavirus testing all year round for stool specimens from gastroenteritis cases in children aged $<5$ years. Enzyme immunoassays are the diagnostic methods of choice for the majority of laboratories, and no major changes in testing policy have occurred since their widespread introduction in 1993.

Weekly counts (by date the stool specimen arrives at the laboratory) of laboratory-confirmed rotavirus reports from children aged $<5$ years born between 1998 and 2007 were extracted from the national database. Before 1998 there was limited recording of date of birth, so children born before 1998 were excluded from this analysis. Numbers of monthly live births in England and Wales during this period were obtained from the Office for National Statistics [8].

Season of birth was divided into winter (December, January, February), spring (March, April, May), summer (June, July, August) and autumn (September, October, November). Seasonal birth cohorts for each year of birth between 1998 and 2007 were formed by combining all the live births occurring in each season. For each birth cohort, the risk of rotavirus diarrhoea at age $m$ months was calculated by dividing the number of rotavirus reports in children of age $m$ born in a given season and year, by the number of live births in the corresponding birth cohort. Cumulative risks for each birth cohort up to age 5 years were calculated. Poisson regression models were fitted to estimate the effect of birth season on risk of rotavirus diarrhoea. The measure of effect used was the relative risk (RR). The population attributable risk fraction (PAF) was calculated to estimate the proportion of all cases in the study population that could be attributed to being born in the summer months.

The formula used was:

$\mathrm{PAF}=\frac{p(\mathrm{RR}-1)}{\mathrm{RR}}$,

where $p$ is the proportion of reported cases that occurred in children born in the summer months and $\mathrm{RR}$ is the relative risk.

There were 95837 reports of laboratory-confirmed rotavirus infections in children aged 0-4 years born between 1998 and 2007. Of these cases $55 \%$ were male, and the highest rate of laboratory reported disease was in children aged $<1$ year $(9 \cdot 2 / 1000$ personyears) (Table 1). Rotavirus reports showed marked seasonality during the study period. The annual 
Table 2. Age-stratified season of birth-specific risk of reported laboratory-confirmed rotavirus infections in children aged $0-4$ years in England and Wales (1998-2007)

\begin{tabular}{|c|c|c|c|c|}
\hline Variable & Winter & Spring & Summer & Autumn \\
\hline \multicolumn{5}{|l|}{ Age (yr) } \\
\hline 0 & 1 & $1.96(1.90-2.02)$ & $2 \cdot 13(2 \cdot 07-2 \cdot 19)$ & $1 \cdot 44(1 \cdot 40-1 \cdot 49)$ \\
\hline 1 & 1 & $0.76(0.74-0.78)$ & $0.73(0.71-0.75)$ & $0 \cdot 88(0 \cdot 86-0.91)$ \\
\hline 2 & 1 & $0.76(0 \cdot 72-0 \cdot 80)$ & $0.67(0.63-0.70)$ & $0.78(0 \cdot 74-0.82)$ \\
\hline 3 & 1 & $0.64(0 \cdot 58-0 \cdot 70)$ & $0.55(0.50-0.60)$ & $0.61(0.55-0.67)$ \\
\hline 4 & 1 & $0.92(0 \cdot 79-1 \cdot 08)$ & $0.83(0.70-0.97)$ & $0.93(0 \cdot 80-1 \cdot 09)$ \\
\hline $\begin{array}{l}\text { Cumulative } \\
\text { relative risk } \\
\text { to age } 5 \mathrm{yr}\end{array}$ & 1 & $1 \cdot 13(1 \cdot 11-1 \cdot 15)$ & $1 \cdot 15(1 \cdot 13-1 \cdot 17)$ & $1 \cdot 03(1 \cdot 01-1 \cdot 05)$ \\
\hline
\end{tabular}

Values given are relative risk ( $95 \%$ confidence interval).

rotavirus season began in November, peaked between February and April and returned to baseline by June. Sixty-five to $70 \%$ of all reports occurred between February and April each year.

Male gender, age and season of birth were associated with an increased risk of reported rotavirus diarrhoea (Table 1). In their first year of life, children born in summer had the highest risk of reported disease (12.0/1000 live births), which was more than double that observed for winter births (RR 2.13, $95 \%$ CI 2.07-2.19) (Table 2). However, in the second and subsequent years, summer-born children had a lower risk than those born in winter (Table 2). Despite the lower risk of disease from the second to fifth year of life, children born in summer had a higher cumulative risk of disease by age 5 years (RR $1 \cdot 15,95 \%$ CI $1 \cdot 13-1 \cdot 17)$ compared with winter births (Table 2 ). All the excess relative risk for summer births occurred during the first year of life. Similar observations were seen for children born in spring and autumn compared with winter births, but with more moderate effects.

We have found evidence that season of birth partly determines risk of rotavirus disease, particularly in the first year of life. These findings are probably explained by an interaction between the seasonality of the disease in England and Wales and the immunological factors that determine susceptibility and protection to infection.

Children born in winter encounter their first rotavirus season aged $0-3$ months. At this age, they are at low risk of developing disease because of high levels of maternal antibody, breastfeeding and limited contact with infectious individuals. Further, if infection does occur at this early age, it is often asymptomatic but still confers some protection against subsequent rotavirus infection and disease [6]. Those born in spring and summer encounter their first rotavirus season aged about 6-11 months, at around the age when maternal antibody levels wane, breastfeeding stops and the potential for exposure to rotavirus is greater due to increased social interaction and increased oral-faecal contact. Thus, in their first year of life, spring and summer births reach the period in the year of highest virus circulation at an age of high exposure, and low maternal antibody protection. The finding that spring and summer births have a lower risk of clinical disease in subsequent years compared with winter births supports previous observations of the protection offered by natural rotavirus infection against subsequent severe disease [9]. Winter-born children have the highest risk of clinical disease in the second year of life because the protection afforded by maternal antibody during their first rotavirus season delays the average age of disease in this group.

Summer birth accounted for $3.6 \%$ (PAF $3.6 \%$, $95 \%$ CI $3 \cdot 2-4 \cdot 0)$ of reported laboratory-confirmed rotavirus infections in our study population. During the study period, the average annual number of reports of laboratory-confirmed rotavirus diarrhoea in <5-year-olds in England and Wales was 13431. Thus, if the risk of reported rotavirus disease in summer-born infants was the same as that in winterborn infants, an average of 484 reported cases would be prevented.

This analysis is predicated on the assumption that seasonal patterns in incidence of reported, laboratoryconfirmed rotavirus infections are representative of seasonal patterns of rotavirus diarrhoea in the population. Reports of laboratory-confirmed rotavirus 
infections represent only a fraction of cases occurring in the community as only a proportion seek medical attention and, of these, stool specimens are investigated for only a fraction [1]. It has been estimated that for every rotavirus case reported to national surveillance there are 1.5 laboratory investigations, 11.3 cases who present to general practice, and 35 community cases [1]. If rotavirus testing is less likely to be offered by laboratories outside of the winter months, and summer births are more likely to be infected in winter months during their first year of life, then our analysis could overestimate the frequency of reported disease in summerborn relative to winterborn infants. Further, disease severity is a strong determinant of seeking medical attention [10]. As a result these cases are more likely to have stool specimens sent and rotavirus-positive specimens reported. If severe disease is more common in children aged $<12$ months and disease is more common in summer births only in this age group, the season of birth difference in cumulative risk may partly be an artefact of higher reporting of cases in the $<1$-year group. However, even if winter births do not have overall lower rates of disease, they would tend to have less severe disease, since infections would occur at an older age when risk of severe disease is lower.

The association between season of birth and risk of rotavirus diarrhoea is an important finding. In England and Wales, a successful rotavirus vaccination programme aimed at reducing transmission and disease incidence would need to ensure high coverage rates of spring and summer births. This is because those born in spring and summer are likely to have an important role in transmission of the virus, as they are at higher risk of diarrhoea during their first year of life, and as a consequence are more likely to be infectious to others. Protecting all infants should be the ultimate goal of a vaccination programme, but season of birth effects should be considered. If good coverage can be rapidly achieved in spring and summer births prior to exposure to their first rotavirus season, the programme may have a more immediate impact and a higher initial effectiveness.

These results are likely to be applicable to other temperate countries where rotavirus gastroenteritis is highly seasonal. To date, no other studies have directly addressed this question. Similar studies in other settings, and in settings with different seasonal patterns of rotavirus diarrhoea, would help better define the role of season of birth in determining risk of rotavirus disease.

\section{ACKNOWLEDGEMENTS}

This work was supported by a grant from the Medical Research Council to Dr C. J. Atchison. The views and opinions expressed in this paper do not necessarily reflect those of the funding body.

\section{DECLARATION OF INTEREST}

None.

\section{REFERENCES}

1. Wheeler JG, et al. Study of infectious intestinal disease in England: rates in the community, presenting to general practice, and reported to national surveillance. The Infectious Intestinal Disease Study Executive. British Medical Journal 1999; 318: 1046-1050.

2. Tompkins DS, et al. A study of infectious intestinal disease in England: microbiological findings in cases and controls. Communicable Disease and Public Health 1999; 2 : 108-113.

3. Sethi D, et al. A study of infectious intestinal disease in England: risk factors associated with group A rotavirus in children. Epidemiology and Infection 2001; 126: $63-70$

4. Koopmans M, Brown D. Seasonality and diversity of Group A rotaviruses in Europe. Acta Paediatrica (Suppl.) 1999; 88: 14-19.

5. Van Damme $\mathbf{P}$, et al. Distribution of rotavirus genotypes in Europe, 2004-2005: the REVEAL Study. Journal of Infectious Diseases 2007; 195 (Suppl. 1): S17-25.

6. Offit PA. Correlates of protection against rotavirus infection and disease. Novartis Foundation Symposium 2001; 238: 106-113.

7. World Health Organisation. WHO position paper on rotavirus vaccines. Weekly Epidemiological Record 2007; 82: 285-296.

8. Office for National Statistics. Birth Statistics - FM1 (Historical Series) (http://www.statistics.gov.uk/statbase). Accessed 1 June 2008.

9. Velazquez FR, et al. Rotavirus infections in infants as protection against subsequent infections. New England Journal of Medicine 1996; 335: 1022-1028.

10. Tam CC, Rodrigues LC, O'Brien SJ. The study of infectious intestinal disease in England: what risk factors for presentation to general practice tell us about potential for selection bias in case-control studies of reported cases of diarrhoea. International Journal of Epidemiology 2003; 32: 99-105. 